\title{
Mechanical and morphological characterization of PMMA/bone composites in human femoral heads
}

\author{
Amelie Sas ${ }^{1}$, Benedikt Helgason ${ }^{2}$, Stephen J. Ferguson ${ }^{2}$, G. Harry van Lenthe ${ }^{1}$ \\ ${ }^{1}$ Biomechanics Section, Department of Mechanical Engineering, KU Leuven, Leuven, Belgium \\ ${ }^{2}$ Institute for Biomechanics, ETH Zurich, Zurich, Switzerland
}

\begin{abstract}
Author contributions:
Amelie Sas: Conceptualization, Methodology, Software, Validation, Formal Analysis, Investigation, Writing - Original draft, Visualization, Project Administration, Funding Acquisition.

Benedikt Helgason: Conceptualization, Methodology, Investigation, Writing - Review \& Editing, Supervision, Funding Acquisition.

Stephen J. Ferguson: Conceptualization, Methodology, Resources, Writing - Review \& Editing, Supervision, Funding Acquisition.

G. Harry van Lenthe: Conceptualization, Methodology, Resources, Writing - Review \& Editing, Supervision, Funding Acquisition.
\end{abstract}

\section{Corresponding author:}

Harry van Lenthe

Biomechanics Section, KU Leuven, Celestijnenlaan 300C, 3001 Leuven, Belgium

harry.vanlenthe@kuleuven.be T +321632 2595

Declarations of interest: None 


\section{Abstract}

2 PMMA has gained an important place in a variety of orthopaedic applications in the femur. However, appropriate data on the mechanical properties of bone-cement composites from the human femur are

4 lacking. Therefore, the goal of this study was to determine the morphological and quasi-static compressive 5 properties of proximal femoral bone-cement composites. Thirty trabecular bone specimens were 6 extracted from fifteen pairs of human femoral heads using specimen-specific cutting guides to ensure an 7 accurate alignment with the main trabecular direction (MTD). One specimen from each pair was 8 augmented with PMMA bone cement, while the other one was left untreated. Specimens were scanned 9 with $\mu \mathrm{CT}$ to determine morphological parameters and tested in quasi-static compression until failure. We found that the long axis of the specimens was highly aligned with the MTD (mean error $<5^{\circ}$ ). A higher

11 compressive modulus and ultimate strength were observed for the bone-cement composite specimens (E

$\left.12=5.7 \pm 0.4 \mathrm{GPa} ; \sigma_{u}=77.9 \pm 5.1 \mathrm{MPa}\right)$ compared to the bone only specimens $\left(\mathrm{E}=2.9 \pm 0.7 \mathrm{GPa} ; \sigma_{u}=19.0\right.$ $13 \pm 5.8 \mathrm{MPa}$ ). Furthermore, the composites had a higher modulus, but lower strength than cement itself (E $14=5.0 \pm 0.3 \mathrm{GPa} ; \sigma_{u}=85.9 \pm 2.7 \mathrm{MPa}$ ) and the composite modulus was significantly correlated with the 15 bone volume fraction (BV/TV). These results are in contrast to previous findings on human vertebral bone, 16 where the composite was more compliant than cement and no correlation was found between BV/TV and 17 the composite modulus. Thus, properties of bone-cement composites cannot simply be applied across 18 different anatomical sites; the site-specific differences in bone density and trabecular alignment should 19 be taken into account. Collectively, the present results suggest that at low BV/TV, cement dominates the composite properties, while at high BV/TV, the contribution of bone becomes apparent, revealing a

21 positive relationship between BV/TV and the on-axis modulus.

22 Keywords: Femur, Bone, Cement, PMMA, Material properties 


\section{Introduction}

Poly(methyl methacrylate) (PMMA) bone cement was introduced in 1960 for the fixation of a femoral hip

prosthesis [1]. Ever since, PMMA has gained an important place in a variety of applications in orthopaedic and trauma surgery [2]. PMMA is an inert material that consists of two components, a liquid and a powder, that are mixed in the operating room to form a dough-like material which is then applied to the bone and subsequently hardens to provide mechanical stabilization of the bone and/or prosthesis.

In the femur specifically, PMMA is currently used in applications such as total hip arthroplasty, fracture

fixation and femoroplasty of metastatic bone lesions. These treatments are often modelled with finite element (FE) analysis for the purpose of evaluating the mechanical effect and/or optimizing the treatment method $[3,4]$. A variety of composite bone-cement material properties have been assumed in these $\mathrm{FE}$ studies, ranging from those of bone cement [4-6], to those of bone-cement composite based on results from vertebral bone studies [7] and to the summed stiffness of bone and cement [8]. It remains to be determined whether these assumptions are appropriate for modeling the behavior of augmented bone in the proximal femur, because accurate knowledge on the material properties of PMMA/bone composite

37 in human femoral bone is lacking.

Thus far, the compressive behavior of PMMA/bone composites has been investigated mainly in the context of vertebroplasty in spinal surgery [9-12]; all these studies have made use of specimens from human vertebral or bovine tibial bone, which differ from human femoral bone in both density and structure. These site-specific differences in bone structure are well known and have already been shown

42 to influence the material properties of human trabecular bone $[13,14]$. It is very likely that these 43 differences also influence the cement distribution and mechanical behavior of bone-cement composites. 44 Indeed, conflicting findings in literature regarding composite stiffness suggest that this is the case. 45 Specifically, several studies on human vertebral PMMA composites found no influence of the bone volume 46 fraction (BV/TV) on the material properties and observed a significantly lower stiffness for bone-cement 
47 composite compared to cement itself $[9-11,15]$. In contrast, for bovine tibial bone, Williams and Johnson

48 found a strong correlation between BV/TV and the modulus in the direction of major trabecular 49 alignment, and their composites showed a higher compressive stiffness compared to cement [16]. These 50 data imply that the results based on human vertebral bone might not apply across different anatomical 51 sites with a higher BV/TV, such as the proximal femur [9].

52 Another important aspect that can influence the material properties of bone-cement composites is the 53 alignment of the specimens with the main trabecular direction (MTD). Off-axis alignment of approximately $5420^{\circ}$ can already result in $40 \%$ difference in the mechanical properties of native trabecular bone in the 55 femoral head [17]. Furthermore, specimen alignment has been shown to influence the mechanical properties of PMMA/bone composites $[12,16]$. The observations of Williams and Johnson, pertaining to 57 the composite modulus being correlated with BV/TV and being higher than the cement modulus, only 58 held true when testing along the MTD and not when testing in the transverse directions [16]. Additionally, 59 Helgason et al. [12] suggested that the reason they did not observe a strong correlation between BV/TV and the composite modulus in bovine tibial specimens was due to the large variation in alignment of their 61 specimens. Thus, there is a scope for developing methods that may be used to prepare bone and bone62 based composite specimens with an accurate alignment with the MTD. In turn, this should contribute to 63 the accurate determination of on-axis material properties and will likely result in stronger correlations 64 between BV/TV and the mechanical response of bone-cement composites $[12,17]$.

65 The purpose of the present study was to determine morphological and quasi-static compressive 66 properties of PMMA/bone composites in human femoral heads. More specifically, the purposes were (1) 67 to develop a method for extracting proximal femoral bone specimens with an accurate on-axis alignment 68 with the MTD, (2) to compare the on-axis compressive stiffness, strength and energy to failure of proximal 69 femoral bone-cement composites to those of native femoral bone and cement, and (3) to determine the 70 influence of bone and cement morphological parameters on these compressive properties. 


\section{2. Materials and Methods}

72 Fifteen pairs of fresh-frozen $\left(-20^{\circ} \mathrm{C}\right)$ human cadaveric femoral heads were obtained from the Department

73 of Anatomy KU Leuven (age 66-98 years, 11 males and 4 females) through their Body Donation Program.

74 Only adult specimens without any abnormalities were included. Ethical approval was granted by the Ethics

75 Committee of the University Hospitals Leuven (reference number NH019 2019-06-01).

\subsection{Design of cutting guides}

77 From each femoral head, a cylindrical trabecular bone specimen was extracted with its axis aligned with

78 the main trabecular direction (MTD). To assure a precise on-axis location of the cylindrical specimen,

79 cutting guides were prepared using additive manufacturing [18] (Fig. 1). Specifically, computed

80 tomography (CT) scans of the femoral heads were acquired (Siemens Somatom Force, Siemens AG,

81 Germany) to determine the desired location of the specimen. The following scanner settings were used:

82120 ref kV, 250 ref mAs, slice thickness $0.4 \mathrm{~mm}$, slice increment $0.2 \mathrm{~mm}$, pitch 0.85 , bone kernel, in-plane

83 pixel size $0.4 \mathrm{~mm}$. The CT scans were imported into Mimics 22.0 (Materialise NV, Leuven, Belgium) and

84 the MTD was identified by the operator through visual inspection of two orthogonal planes (Fig. 1A). A

85 virtual cylinder representing the specimen was positioned on the scans such that its axis was aligned with

86 the MTD. Perpendicular to the MTD, a plane was drawn to indicate the position for cutting the specimen

87 such that inclusion of cortical bone was prevented. The surface contour of the femoral head was

88 segmented from the CT scan and converted into a 3D solid part. Using the femoral head geometry,

89 together with the position of the cylinder and the cutting plane, a specimen-specific cutting guide was

90 designed in 3-matic 14.0 (Materialise NV, Leuven, Belgium) (Fig. 1B). The cutting guide consisted of two

91 parts, each with a concave side that fitted onto the femoral head. The circular opening at the top and the

92 cutting slot on the sides indicated the coring direction and the cutting position respectively. The parts 

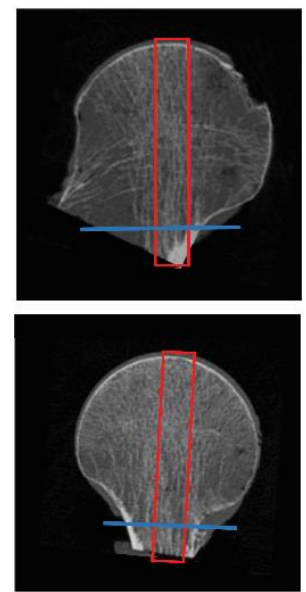

A
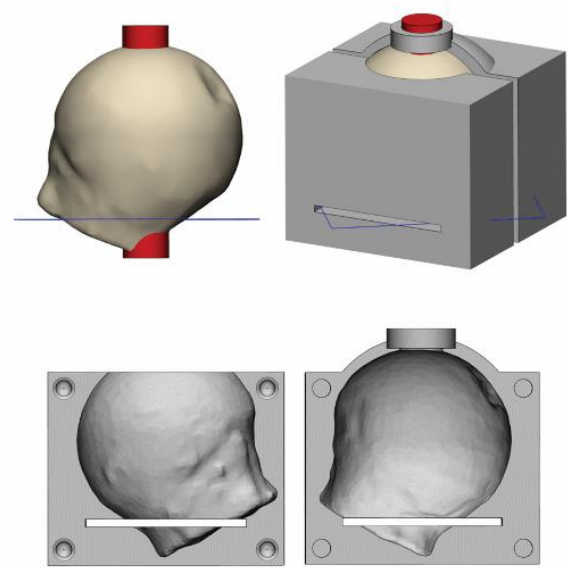

B
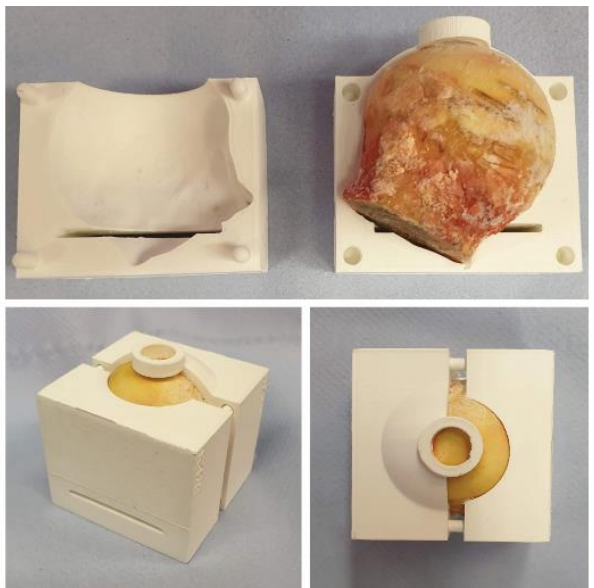

C

Fig. 1: Procedure for manufacturing a specimen-specific cutting guide to assure specimen extraction along the main trabecular direction (MTD). (A) A cylinder representing the specimen (red) was positioned on the CT scan such that it was aligned with the MTD and a cutting plane (blue) was defined to eliminate cortical bone within the specimen. (B) Based on these references, a specimen-specific cutting guide was designed that indicated the coring direction and cutting position. The guide consisted of two parts that fitted to the shape of the femoral head. (C) The cutting guides were additively manufactured and assembled on the femoral heads.

\subsection{Preparation of bone specimens}

After assembling the specimen-specific cutting guides on the femoral heads (Fig. 1C), cylindrical bone specimens were cored along the indicated direction using a diamond-coated coring tool (BREU

distilled water until a depth just below the cutting plane. Subsequently, the specimens were cut along this

Germany). Continuous cooling was provided with distilled water. 
in a smooth soap solution [10]. Subsequently, the specimens were placed with the soap solution in closed

108 vials inside an ultrasonic water bath for $20 \mathrm{~min}$ (VWR International Ltd., Lutterworth, UK), and afterwards

109 rinsed with a high-pressure pulse lavage system (Palavage Heraeus Holding GmbH, Hanau, Germany). This

110 process of ultrasonic and water jet cleaning was repeated at least two times until complete marrow

111 removal was visually confirmed.

112 After cleaning, each specimen was submerged in phosphate buffered saline (PBS) solution and scanned

113 inside a $\mu C T$ scanner ( $\mu C T$ 100, Scanco Medical AG, Bassersdorf, Switzerland) to obtain bone

114 morphological parameters. The following scanner setting were used: $70 \mathrm{kVp}, 114 \mu \mathrm{A}, 14.8 \mu \mathrm{m}$ voxel size, $115200 \mathrm{~ms}$ integration time and $0.5 \mathrm{~mm}$ aluminium filter.

116 After scanning, the length and the diameter of the specimens were measured with a digital caliper

117 (Mettler Toledo, Greifensee, Switzerland, accuracy $\pm 0.01 \mathrm{~mm}$ ). Finally, the specimens were dried on a 118 napkin for a short time and wrapped in PBS soaked bandage for storage in separate plastic bags in a 119 freezer at $-20^{\circ} \mathrm{C}$ until the day of augmentation. In total, 30 bone specimens were successfully prepared.

\subsection{Cement augmentation}

121 The paired specimens were divided in two groups such that one specimen of each pair was randomly 122 assigned for cement augmentation (composite group), while the other was left untreated (bone group).

123 Additionally, 15 cement only specimens were prepared (cement group). All bone specimens were thawed 124 overnight in a refrigerator at $4^{\circ} \mathrm{C}$ before the day of cement augmentation.

125 The composite and cement specimens were prepared using a polytetrafluoroethylene (PTFE) split-mold 126 [19] (Fig. 2). For preparation of the composite specimens, a bone cylinder was positioned at the center of 127 the mold before assembling. A copper mesh was placed between the mold and the bottom plate to allow 128 air to escape during cement injection (Fig. 2A). The PMMA bone cement (Traumacem V+, Synthes GmbH, 129 Oberdorf, Switzerland) was prepared by hand-mixing the powder and liquid components with a metal spatula for $30 \mathrm{~s}$. The cement was then transferred into a $5 \mathrm{ml}$ syringe with the tip cut off and immediately 
131 injected into the bone cylinder via the top opening of the PTFE mold (Fig. 2B). The injection was stopped

132 when cement visibly penetrated the copper mesh at the bottom end of the molds. After $1 \mathrm{~h}$ of initial 133 setting at room temperature, the augmented bone specimens were removed from the molds. The ends 134 were trimmed to the desired specimen length $(26 \mathrm{~mm})$ with the diamond-coated bandsaw. Diameter and 135 length were measured with a digital caliper.

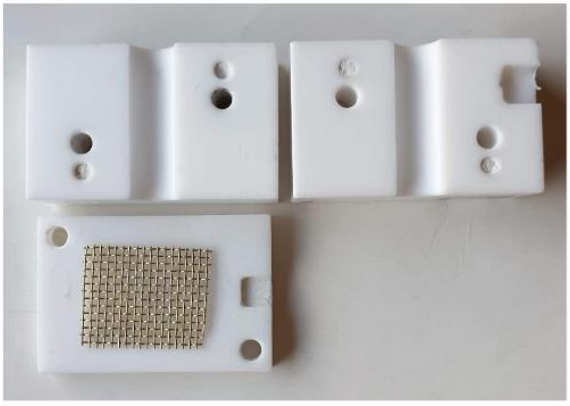

A

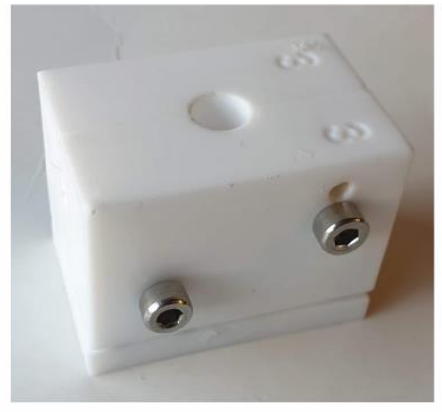

B

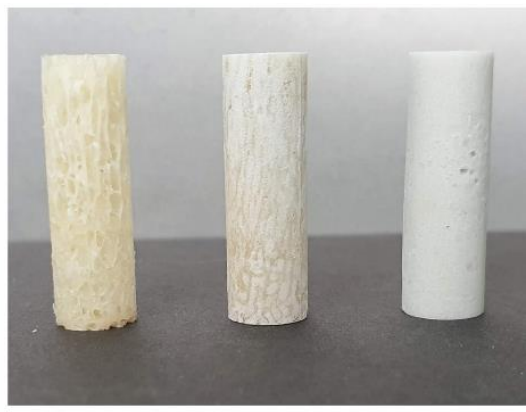

C

Fig. 2: PTFE split-molds used for cement augmentation. (A) Disassembled split-mold; for preparation of the composites, a bone specimen was placed at the center of the mold before assembly. (B) Assembled split-mold. (C) From left to right an example of a native bone specimen, a composite specimen and a cement only specimen.

136 The specimens in the cement group were prepared with a similar procedure. In this case, cement was

137 injected into an empty split-mold. The only difference in the procedure was a $30 \mathrm{~s}$ agitation on a vibration plate (KV-26 plus, Wassermann Dental-Maschinen GmbH, Hamburg, Germany) immediately after cement injection to remove large entrapped air holes.

All specimens were stored in individual plastic containers filled with PBS solution and left to set for approximately $24 \mathrm{~h}$ at $37^{\circ} \mathrm{C}$. After setting of the cement, the specimens from the composite and cement group were scanned using $\mu \mathrm{CT}$ for quantifying cement porosity. Finally, aluminum endcaps (cap depth 5 $\mathrm{mm}$ ) were glued onto the specimens using an epoxy-based adhesive (Araldit Rapid, Araldite, Basel, Switzerland) to minimize structural end-effects [20]. Alignment of the end caps during gluing was controlled by fixing the capped specimens in a custom-made alignment holder. The final dimensions of the exposed bone between the end caps was $8 \mathrm{~mm}$ in diameter and $16 \mathrm{~mm}$ in height (aspect ratio $=2$ ). In between processes, the specimens were returned to a container with PBS at room temperature until mechanical testing. 


\subsection{MicroCT analysis}

150 The $\mu \mathrm{CT}$ images of the native bone specimens were analyzed to quantify bone morphological parameters 151 and specimen alignment with the MTD. The images were Gaussian filtered (sigma = 1.2, support $=1$ ) and 152 segmented using a fixed global threshold of $22 \%$ of the maximum grey scale value (corresponding to 485 $153 \mathrm{mg} \mathrm{HA} \mathrm{cm}{ }^{-3}$ according to a phantom calibration) [21] (Fig. 3A). Within a cylindrical volume of interest (VOI) 154 of diameter $7 \mathrm{~mm}$ and height $16 \mathrm{~mm}$, the following bone morphological parameters were quantified: bone 155 volume fraction (BV/TV), trabecular number (Tb.N), trabecular thickness (Tb.Th), trabecular separation 156 (Tb.Sp), structure model index (SMI), connectivity density (Conn.D), and degree of anisotropy (DA). 157 Trabecular orientation was assessed by calculating the mean intercept length (MIL) [22]. The MTD 158 direction of the cylindrical bone specimen was defined as the direction of the eigenvector corresponding 159 to the maximum eigenvalue of the MIL tensor. The misalignment of the specimen axis from this MTD (off160 axis angle) was defined as the angle between the MTD and the long axis of the cylindrical specimen.

161 The $\mu \mathrm{CT}$ images of the composite and cement specimens were analyzed to quantify the pore volume 162 fraction (PV/TV). A Gaussian filter with a higher support level (sigma $=1.2$, support $=5$ ) was applied since 163 the heavy zirconium metal increased the amount of image noise. The porous domain was obtained by 164 segmenting the bone and cement together with a fixed threshold value determined by visual inspection 165 (28\% of maximum grey value, $670 \mathrm{mg} \mathrm{HA} \mathrm{cm}^{-3}$ ) (Fig. 3B-C). Within the cylindrical VOI (diameter $7 \mathrm{~mm}$, 166 length 16), the combined bone and cement volume fraction (BV+CV)/TV was then quantified and PV/TV 167 was obtained by assuming that PV/TV $=1-(\mathrm{BV}+\mathrm{CV}) / \mathrm{TV}$. In case of the cement only specimens (Fig. 3C), 168 no bone was present and $\mathrm{BV}$ in the previous formula was equal to zero. 

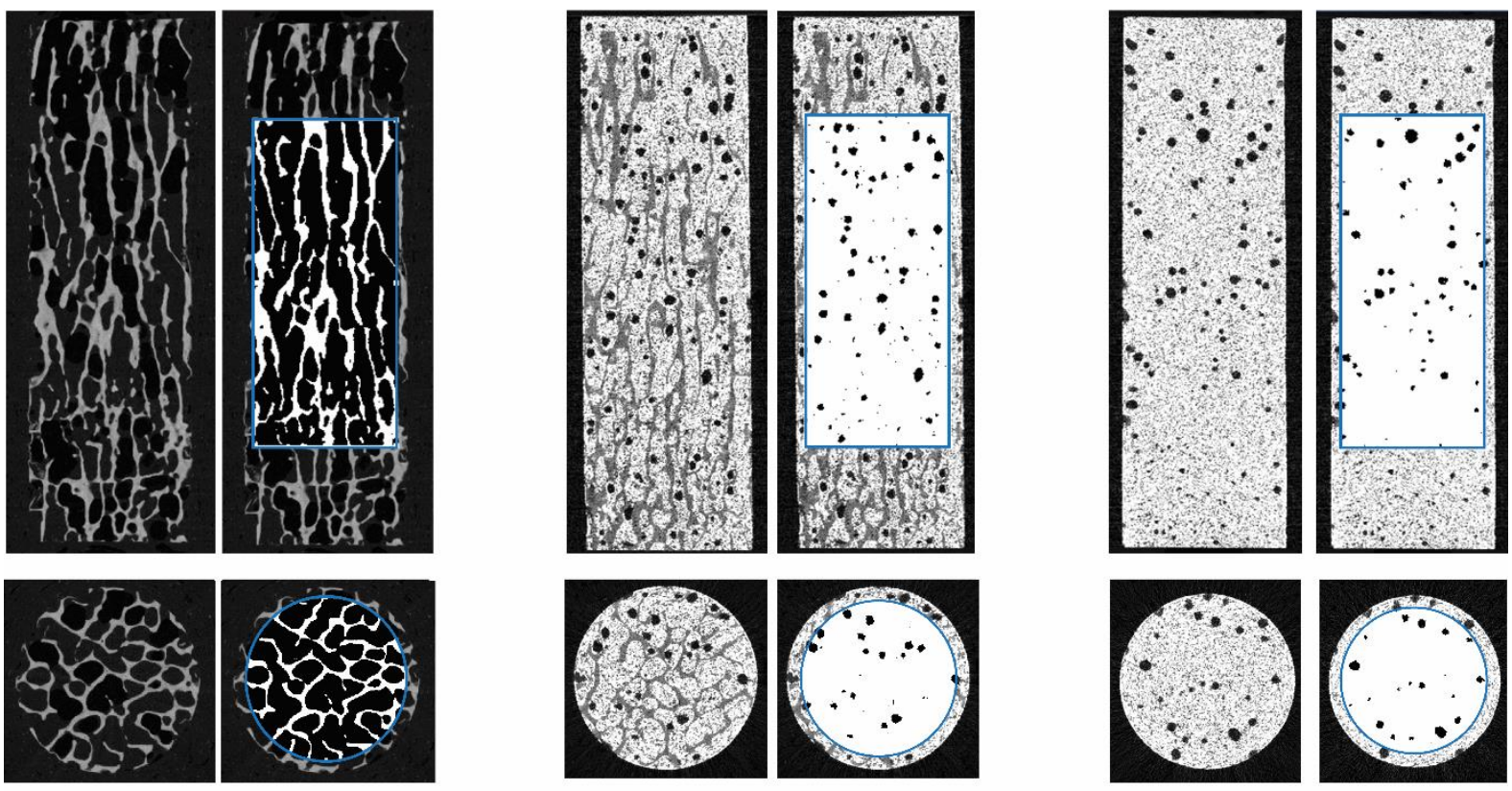

A

$\mathrm{B}$

$\mathrm{C}$

Fig. 3: Segmentation of the $\mu C T$ scans within the cylindrical VOI (blue contour). A) Bone was segmented from the native bone scans to extract bone morphological parameters. B) Bone and cement were segmented together from the composite scans to quantify the pore volume fracture (PV/TV). C) Cement was segmented from the cement only scans to quantify the PV/TV.

\subsection{Quasi-static compression testing}

170 Capped bone, composite and cement specimens were tested to failure in compression on an Instron

171 E10000 materials testing machine (Instron, High Wycombe, UK). To avoid the influence of machine

172 compliance and end-artifacts, strain was measured using an extensometer (Model 3442-006M-013-ST,

173 Epsilon Technology Corp., Jackson) with a gauge length of $6 \mathrm{~mm}$ (Fig. 4). Five preconditioning cycles from

1740.1 to $0.4 \%$ strain at a rate of $0.5 \%$ per second [23] were applied before the specimens were loaded to

175 failure. The ramp to failure was applied in displacement control at a displacement rate of $0.13 \mathrm{~mm} / \mathrm{s}$

176 (corresponding to approximately $0.5 \%$ strain per second). Failure was defined as a $100 \mathrm{~N}$ drop in the load

177 as recorded by the load cell [24]. Load, displacement and strain data were acquired at $100 \mathrm{~Hz}$. 


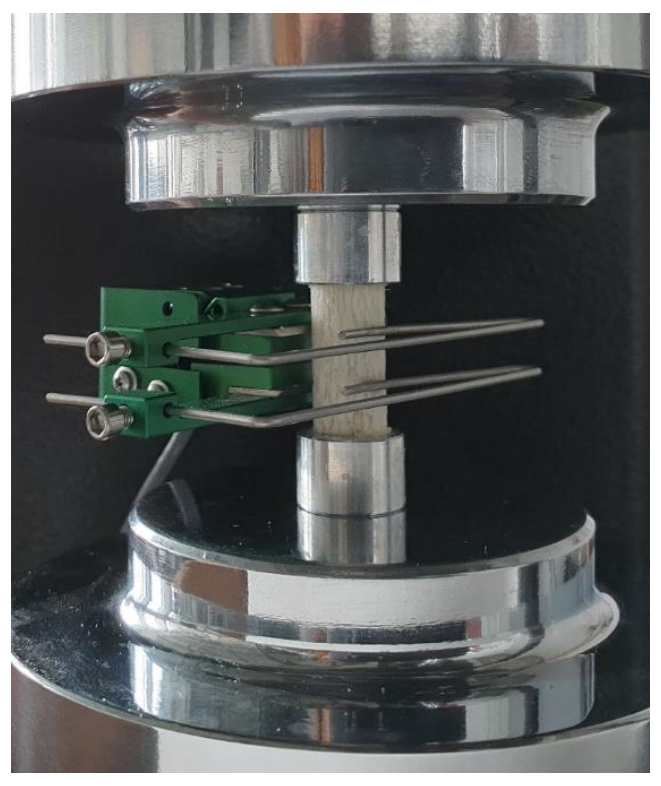

Fig. 4: Mechanical testing setup. Aluminum endcaps were glued onto the specimens. The constructs were tested to failure under compressive loading. Strain was measured with a $6 \mathrm{~mm}$ extensometer attached to the center of the specimen.

Mechanical properties were extracted from the final ramp segment of the compression test results. Stress was defined as the load divided by the nominal cross-sectional area of the specimens. Strain was directly obtained from the extensometer output. The modulus of elasticity (E) was defined as the slope of the stress-strain curve between 0.2 and $0.4 \%$ strain [12]. Yield strength $\left(\sigma_{y}\right)$ and yield strain $\left(\varepsilon_{y}\right)$ were defined by the $0.2 \%$ offset method. Ultimate strength $\left(\sigma_{u}\right)$ was defined as the maximal stress value and energy until failure $(U)$ as the area under the stress-strain curve up to the ultimate strain $\left(\varepsilon_{u}\right)$. $U$ provides a measure of the resistance to fracture [25].

\subsection{Statistical analysis}

Statistical analysis was carried out in Matlab v2020a (Mathworks, Inc., Natick, MA, USA). Statistical

187 differences between groups were identified with Wilcoxon signed-rank tests for comparison of the paired specimens in the bone and composite group, and with Mann-Whitney $\mathrm{U}$ tests for comparison with the cement only group. P-values below 0.05 were considered statistically significant. Linear regression was applied to identify correlations between parameters. The coefficient of determination $\left(R^{2}\right)$ was used to determine the goodness of fit. 


\section{Results}

\subsection{MicroCT analysis}

194 The volume fractions, off-axis angle and bone morphological parameters as measured from the $\mu \mathrm{CT}$ data 195 are listed in Table 1. Statistically significant differences between the bone and composite group were not 196 found for any of the parameters. The off-axis angle was $4.60^{\circ}\left( \pm 2.34^{\circ}\right)$ on average for the bone and 197 composite specimens $(\mathrm{N}=30)$ and never exceeded $10^{\circ}$. Between the composite and cement group, there was no statistical difference in the PV/TV.

Table 1: Summary of the microCT parameters and mechanical properties (mean $\pm S D$; range in parentheses).

\begin{tabular}{|c|c|c|c|}
\hline & Bone $\left(N=14 / 10^{\Delta}\right)$ & Composite $(\mathrm{N}=15)$ & Cement $(N=15)$ \\
\hline \multicolumn{4}{|l|}{ MicroCT parameters } \\
\hline BV/TV (\%) & $27.3 \pm 5.0(18.8-36.8)$ & $26.9 \pm 4.6(20.7-36.6)$ & - \\
\hline CV/TV (\%) & - & $69.7 \pm 4.8(60.3-77.0)^{\S}$ & $97.1 \pm 0.1(95.7-98.0)$ \\
\hline PV/TV (\%) & - & $3.4 \pm 1.0(2.2-5.1)$ & $2.9 \pm 0.1(2.0-4.3)$ \\
\hline Off-axis angle $\left({ }^{\circ}\right)$ & $5.03 \pm 2.34(1.66-9.62)$ & $4.16 \pm 2.33(0.68-8.42)$ & - \\
\hline Conn.D & $4.65 \pm 1.40(2.45-8.18)$ & $4.33 \pm 1.43(2.54-8.43)$ & - \\
\hline SMI & $-0.01 \pm 0.53(-1.20-0.69)$ & $0.03 \pm 0.57(-1.38-0.66)$ & - \\
\hline DA & $2.19 \pm 0.16(1.95-2.50)$ & $2.23 \pm 0.26(1.86-2.69)$ & - \\
\hline Tb.Th & $0.21 \pm 0.03(0.16-0.29)$ & $0.21 \pm 0.03(0.17-0.27)$ & - \\
\hline Th.Sp & $0.68 \pm 0.07(0.54-0.78)$ & $0.70 \pm 0.07(0.59-0.86)$ & - \\
\hline Th.N & $1.35 \pm 0.12(1.21-1.66)$ & $1.31 \pm 0.11(1.08-1.56)$ & - \\
\hline \multicolumn{4}{|l|}{ Mechanical properties } \\
\hline $\mathrm{E}(\mathrm{MPa})$ & $2902 \pm 742(1633-4393)$ & $5715 \pm 417(5106-6403)^{* \S}$ & $4999 \pm 256(4574-5507)^{*}$ \\
\hline$\sigma_{y}(\mathrm{MPa})$ & $20.3 \pm 5.0(12.1-29.5)$ & $50.8 \pm 4.6(41.5-59.6)^{* \S}$ & $57.2 \pm 2.7(53.3-63.9)^{*}$ \\
\hline$\sigma_{u}(\mathrm{MPa})$ & $19.0 \pm 5.8(6.4-29.9)$ & $77.9 \pm 5.1(65.0-86.8)^{* \S}$ & $85.9 \pm 2.7(82.2-91.2)^{*}$ \\
\hline$\varepsilon_{y}(\%)$ & $0.87 \pm 0.04(0.79-0.95)$ & $1.09 \pm 0.05(0.99-1.19)^{* \S}$ & $1.34 \pm 0.04(1.28-1.42)^{*}$ \\
\hline$\varepsilon_{u}(\%)$ & $1.27 \pm 0.26(0.99-1.81)$ & $4.52 \pm 0.79(3.62-6.33)^{*}$ & $4.08 \pm 0.40(3.44-4.78)^{*}$ \\
\hline $\mathrm{U}\left(\mathrm{J} / \mathrm{mm}^{3}\right)$ & $0.18 \pm 0.05(0.11-0.24)$ & $2.75 \pm 0.60(2.08-4.24)^{*}$ & $2.56 \pm 0.30(2.11-3.15)^{*}$ \\
\hline
\end{tabular}

${ }^{\Delta}$ One specimen in the bone group was fully excluded due to preliminary failure during preconditioning. For 4 other specimens, the measurements of $\sigma_{y}, \varepsilon_{y}, \varepsilon_{u}$ and $U$ had to be excluded due to slippage of the extensometer.

* Significantly different $(p<0.05)$ from bone

$\S$ Significantly different $(p<0.05)$ from cement 


\subsection{Mechanical testing}

200 The mechanical test results for one specimen in the bone group were excluded since this specimen already

201 failed during preconditioning. From the remaining 14 specimens in this group, $\sigma_{y}, \varepsilon_{y}, \varepsilon_{u}$ and $U$ could not

202 be obtained for 4 specimens due to slippage of the extensometer prior to failure. In the pairwise

203 comparisons between the bone and composite group, the specimen pairs corresponding to the

204 unavailable measurements were left out.

205 A summary of the mechanical properties for the three groups (bone, cement and composite) is presented

206 in Table 1. Representative stress-strain curves for each group are shown in Fig. 5. All mechanical properties

207 in the bone group were significantly different from both the composite and cement group (Table 1). A

208 higher compressive stiffness, strength, ductility and energy to failure were observed in the composite

209 group compared to the bone group (Table 1 and Fig. 6). When comparing the composite and cement

210 group, the composite specimens showed a significantly higher compressive modulus (E), but a lower

211 strength $\left(\sigma_{y}, \sigma_{u}\right)$ (Table 1 and Fig. 6). The cement specimens had a significantly higher $\varepsilon_{y}$ than the

212 composite specimens, yet no significant differences were observed for $\varepsilon_{u}$ and $U$.

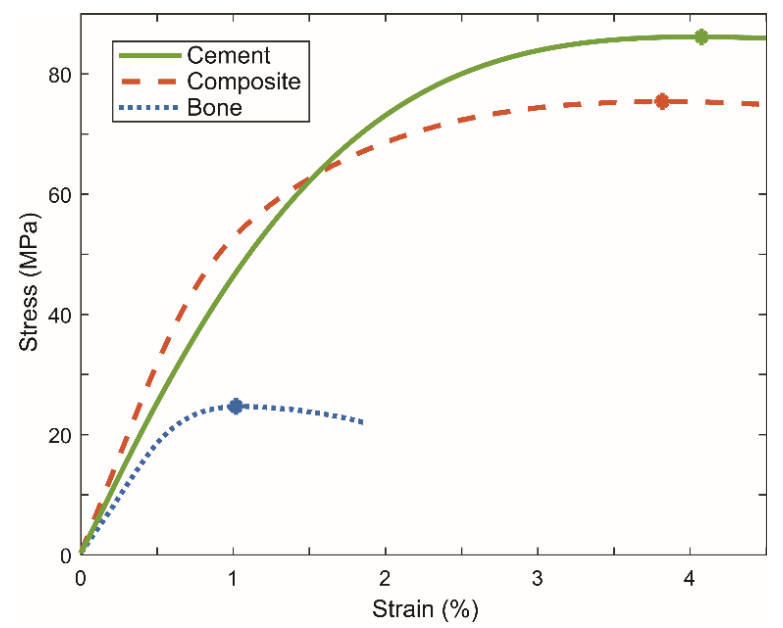

Fig. 5: Representative stress-strain curves for a paired bone and composite specimen, and a cement only specimen. The ultimate strength/strain point is marked with a dot. 

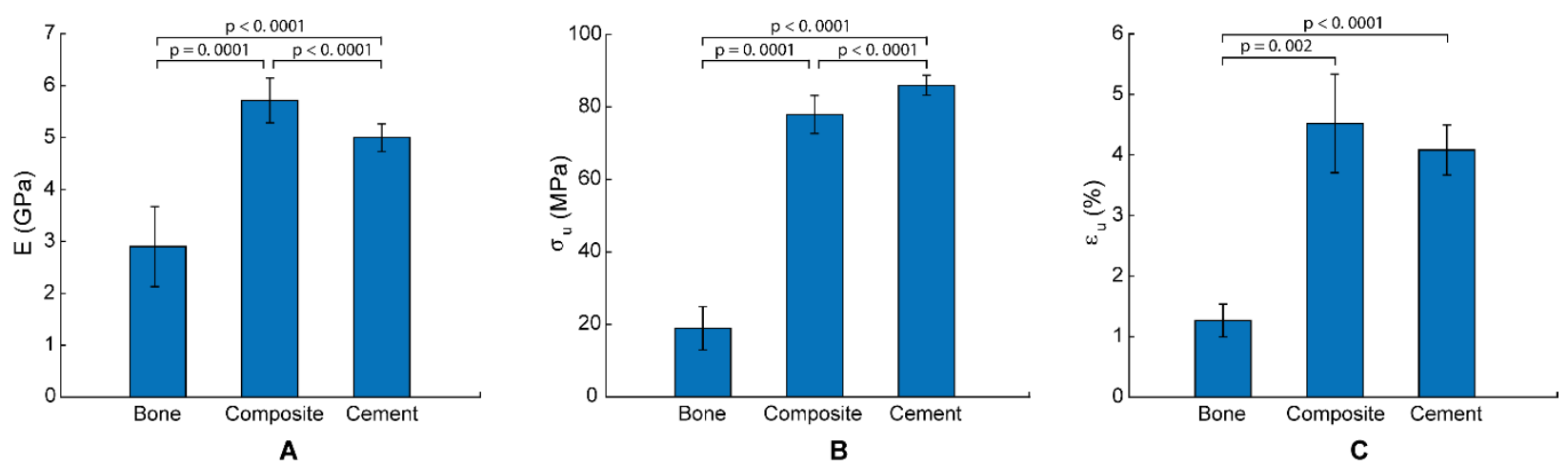

Fig. 6: Comparison of the compressive modulus of elasticity $E(A)$, ultimate strength $\sigma_{u}(B)$ and ultimate strain $\varepsilon_{u}(C)$ between the bone, composite and cement group. Refer to Table 1 for individual groups sizes in the statistical analyses.

\section{$213 \quad 3.3$ Regression analysis}

214 In the bone group, $\mathrm{E}$ and $\sigma_{u}$ showed a strong linear relation to $\mathrm{BV} / \mathrm{TV}\left(R^{2}=0.81\right.$ and 0.96 respectively $)$

215 (Fig. 7A). A similar correlation was observed for $\sigma_{y}\left(R^{2}=0.91\right)$. E, $\sigma_{y}$ and $\sigma_{u}$ were also correlated with SMI and Tb.Th $\left(0.58<R^{2}<0.72\right)$, but the latter two parameters were not independent from BV/TV. SMI and

217 Tb.Th were strongly related to $\mathrm{BV} / \mathrm{TV}\left(R^{2}=0.76\right.$ and 0.73$)$.

218 In the composite group, again a linear relation was found between BV/TV and $\mathrm{E}\left(R^{2}=0.51\right)($ Fig. $7 \mathrm{~B})$.

219 Consequently, E was also correlated with SMI and Tb.Th $\left(R^{2}=0.61\right.$ and 0.65$)$. Yet, in contrast to the bone 220 group, no correlation was found between BV/TV and $\sigma_{y}$ and $\sigma_{u}$ (Fig. 7B). The strength parameters $\sigma_{y}$ and $221 \sigma_{u}$ on the other hand did show a negative relation with the Conn.D $\left(R^{2}=0.53\right.$ and 0.50$)$ (Fig. 8).

222 Furthermore, E also correlated with a cement related parameter CV/TV $\left(R^{2}=0.57\right)$, but the latter was 223 again not independent from BV/TV.

224 Other correlations between the $\mu \mathrm{CT}$ parameters and mechanical properties were not significant or were 225 weak $\left(R^{2}<0.42\right)$. 

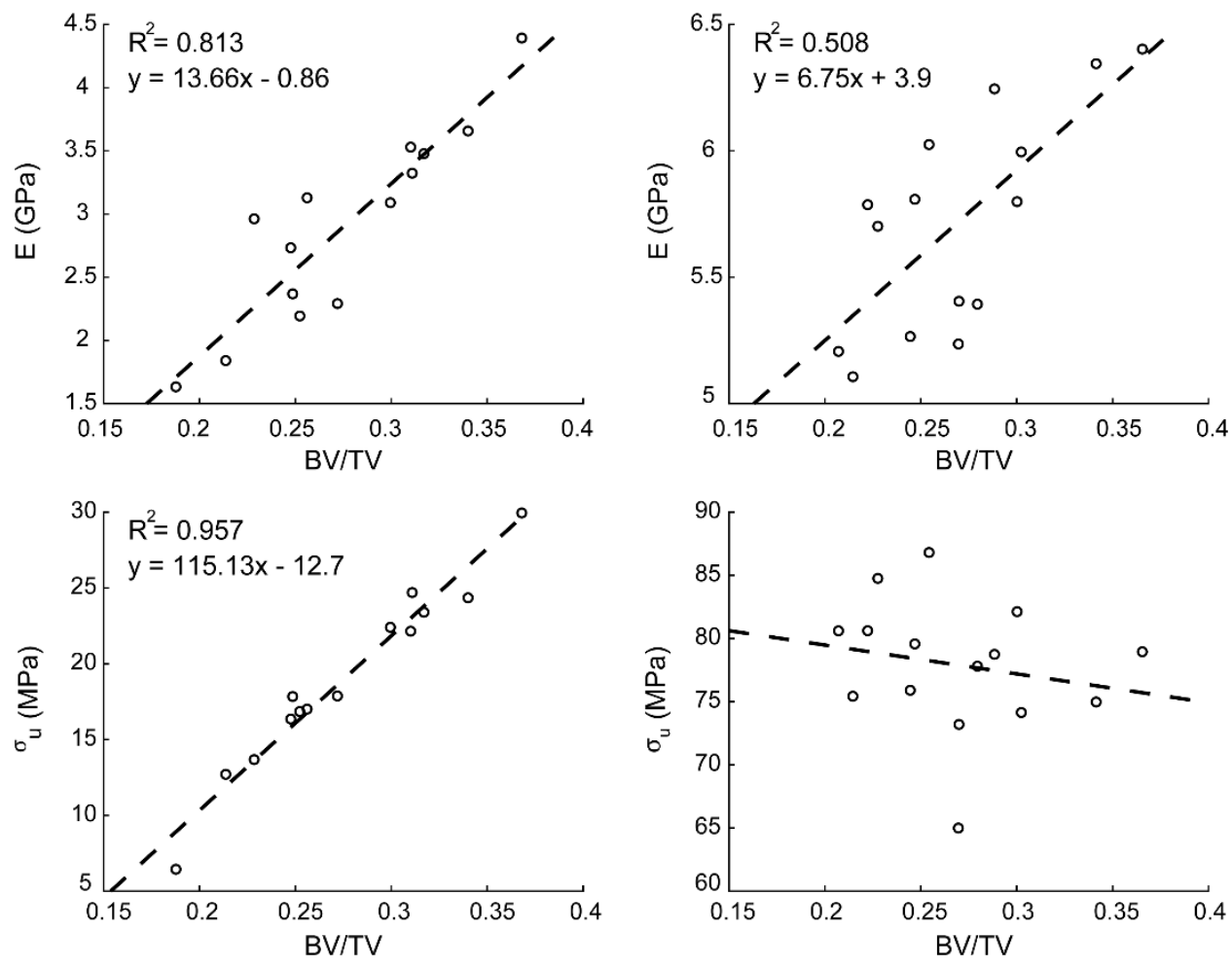

A) Bone

B) Composite

Fig. 7: A) In the bone group, BV/TV showed a strong linear relation with the compressive modulus of elasticity (E) and ultimate strength $\left(\sigma_{u}\right)$. B) In the composite group, BV/TV also showed a significant correlation with $E$, but not with $\sigma_{u}$.
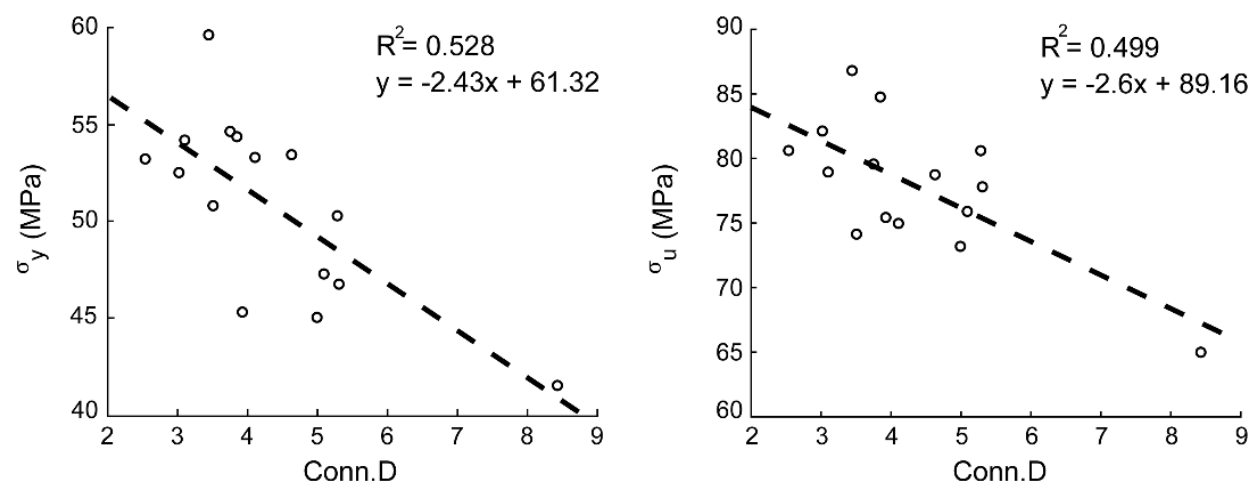

Fig. 8: In the composite group, the yield strength $\left(\sigma_{y}\right)$ and ultimate strength $\left(\sigma_{u}\right)$ were linearly related with the connectivity density (Conn.D).

\section{Discussion}

227 The aim of this study was to characterize the mechanical properties of bone-cement composites produced

228 with trabecular bone specimens retrieved from human femoral heads. These properties can be applied in

229 finite element models to simulate surgical procedures in the human proximal femur that involve cement 
augmentation with PMMA. We found proximal femoral bone-cement composites aligned with the MTD to have a higher compressive modulus, but lower strength than cement only specimens. Furthermore, the composite modulus showed a significant correlation with BV/TV.

An excellent alignment of the specimen axis with the MTD was obtained using specimen-specific 3Dprinted cutting guides. Specimen malalignment was quantified with an off-axis angle of $4.6^{\circ} \pm 2.3^{\circ}$. This error is smaller than the one obtained in previous studies aiming for specimen alignment with the MTD $[17,26-28]$. Using steel markers as a reference, recognizable on CT images to compare the reference axis with the MTD, an off-axis angle of $6.1 \pm 3.3^{\circ}$ was found by Öhman et al. [17] and $7.7 \pm 3.7^{\circ}$ by Perilli et al. [28]. Another study made use of a specimen holder with an adjustable angle such that the position of the specimen before coring the bone cylinder could be accurately adjusted based on a $\mu \mathrm{CT}$ scan. With this method off-axis angles of $5.35 \pm 3.17^{\circ}[26]$ and $6.9 \pm 3.3^{\circ}[27]$ were obtained.

The compressive modulus and strength of the native bone specimens showed a significant correlation with BV/TV, SMI and Tb.Th. The correlations were not independent; SMI and Tb.Th were strongly related to BV/TV. In comparison to the literature $[20,29]$, high coefficients of determination were found for the density-modulus $\left(R^{2}=0.81\right)$ and density-ultimate-strength relationships $\left(R^{2}=0.96\right)$.

245 The compressive modulus of the bone-cement composites was significantly higher than the modulus of 246 bone and cement only specimens. On average, the moduli of bone, composite and cement were $2.9 \pm 0.7$, $2475.7 \pm 0.4$ and $5.0 \pm 0.3 \mathrm{GPa}$ respectively. This result is in agreement with a study on bovine tibial bone [16]; 248 yet, is in contrast to reported data on human vertebral bone, where the composite specimen was more compliant than cement (30-48\% lower) [9-11]. Using a simple rule of mixtures, one would expect a higher modulus for bone-cement composites than for cement since the tissue modulus of trabecular bone (10 -

$25120 \mathrm{GPa}[30,31])$ is higher than the modulus of PMMA cement. In case of perfect bonding between bone 252 and cement, the composite modulus could be calculated as $\mathrm{E}_{\text {composite }}=\mathrm{BV} / \mathrm{TV} \cdot \mathrm{E}_{\mathrm{bone}}+\mathrm{CV} / \mathrm{TV} \cdot \mathrm{E}_{\mathrm{cement}}$ 
253 [16], which would correspond to $6.2-8.9 \mathrm{GPa}$ based on the mean values of our study. However, the

254 modulus we measured was lower, most likely because PMMA cement does not perfectly bond to bone

255 [11]. In addition, some bone damage may have occurred during augmentation [32]. Yet, in the present

256 study we still found the composite modulus to be higher than the modulus for cement alone, while studies

257 on vertebral bone found the modulus of composite specimens to be even lower than for cement alone.

258 An important factor contributing to this difference is the lower BV/TV of vertebral trabecular bone

259 compared to trabecular bone in the femoral head, which reduces the contribution of bone to the

260 composite modulus. In addition, vertebral bone has a different trabecular orientation, which, together

261 with the BV/TV, have been shown to be the main factors influencing the mechanical properties of

262 trabecular bone [29].

263 Similarly to the native bone modulus, the composite modulus was significantly correlated with BV/TV, SMI

264 and Tb.Th. Again, this finding is in disagreement with studies on vertebral bone [9-11], that did not find a

265 significant correlation between the composite stiffness and bone morphological parameters. As discussed

266 in the previous section, this difference can be attributed to the site-specific differences in bone

267 morphology between vertebral and proximal femoral bone. In low-density vertebral bone, cement

268 dominates the mechanical properties, thereby suppressing the role of bone morphology. However, even

269 for high-density bone from the bovine tibia, different findings have been reported [12,16,24]. Helgason

270 et al. [12] found no significant correlation between the composite modulus and BV/TV, Lopez et al. [24]

271 only found a significant correlation between the modulus and BV/TV for low-modulus cement, and

272 Williams and Johnson only found a correlation along the MTD and not in the transverse directions [16].

273 Two other factors thus seem to influence the correlation between the composite modulus and BV/TV,

274 namely the cement modulus and the alignment with the MTD. The effect of the cement modulus was

275 attributed earlier to the increased relative contribution of bone when decreasing the cement modulus

276 [24]. The effect of alignment can be explained similarly; the contribution of bone increases with an 

accurate alignment along the MTD since bone is known to be a lot stiffer along its MTD $[17,29]$. In case no

278 accurate alignment was obtained, the variance caused by different alignments could mask the effect of 279 BV/TV. In the study of Helgason et al. [12], a large variation was observed in the anisotropy ratio, indicating that some of their specimens were tested off-axis. This higher variation in alignment can potentially 281 explain why they did not observe a significant correlation with BV/TV. That same study did find a 282 significant correlation between the degree of anisotropy and the mechanical properties; a correlation we 283 were not able to observe due to the very narrow range in degree of anisotropy. This implies that in order 284 to define the material properties of bone-cement composites across varying directions, not only along the 285 MTD, there will potentially be a need for a modulus-density-morphology relationship that takes into 286 account both the effect of BV/TV and trabecular alignment [29]. In order to define such a relationship, 287 additional testing will be required for specimens across a large spectrum of BV/TV and trabecular 288 architecture.

289 In contrast to the modulus, the composite strength ( $\left.\sigma_{y}=51 \pm 4.6 \mathrm{MPa}, \sigma_{u}=78 \pm 5.1 \mathrm{MPa}\right)$ was found to 290 be lower than the cement strength $\left(\sigma_{y}=57 \pm 2.7 \mathrm{MPa}, \sigma_{u}=86 \pm 2.7 \mathrm{MPa}\right)$, although the trabecular bone 291 tissue strength ( $\sigma_{y}=83 / 134 \mathrm{MPa}$ in tension/compression according to [31]) is higher than the strength of 292 cement. Still, the ratio of bone-to-cement strength is smaller (factor 1.5-2.4) than for the modulus (factor 293 2-4), which makes the relative contribution of bone smaller. In addition, no significant correlations with 294 BV/TV, SMI and Tb.Th were observed. Analogously to the studies on vertebral bone-cement composites, 295 the correlations with bone morphology seem to get masked if cement dominates the composite strength 296 [9-11]. We did observe a negative correlation for $\sigma_{y}$ and $\sigma_{u}$ and the Conn.D, which is a measure of the 297 connectivity of the trabeculae. A possible explanation is that highly connected trabecular bone structures 298 cause more interruptions of the cement structure, which could decrease the strength of the latter. 
299 No correlation with porosity was observed in both the composite and cement group. In contrast, Kinzl et 300 al. did find a strong correlation $\left(R^{2}=0.91-0.98\right)$ between the mechanical properties and porosity, defined 301 as $\mathrm{PV} /(\mathrm{TV}-\mathrm{BV})$ to exclude potentially confounding influences of BV/TV [9]. Also for this porosity 302 definition, no correlations were found in our study, although we believe this might be related to the very 303 low porosity values as opposed to Kinzl et al. (range 3-8\% versus 4-22\%).

304 The focus of this study was on the mechanical evaluation of PMMA bone cement for augmenting proximal 305 femoral trabecular bone, yet recent studies have also shown interest in the development of other 306 functional properties of PMMA. For example, the inclusion of antibiotics as an additive has proven to be 307 useful, although it has its share of shortcomings, including sub-optimal release of the antibiotic and very 308 low effectiveness against bacteria commonly found in cases of periprosthetic joint infection of hip and 309 knee arthroplasties [2,33]. More recently, several nanoparticles with antimicrobial activity have been 310 emerging for reducing bacterial proliferation, i.e. silver and gold [33,34]. In addition to antibacterial 311 activity, gold nanoparticles have also shown to influence the mechanical properties of PMMA, more 312 specifically improving the punching strength of PMMA [34].

313 There are a number of limitations of our study. First, the augmentation was carried out in vitro after 314 thorough removal of bone marrow. This protocol does not present the clinical situation where cement 315 pushes marrow out of the cancellous bone pore space during the injection of the cement [12]. The study 316 by Kinzl et al. [9] addressed this issue by injecting bone cement in a human vertebra without removing 317 the bone marrow in advance. They observed a higher PVF than in our study and they reported that the 318 compressive modulus and yield strength linearly decreased with increasing porosity. The injection into 319 marrow-free cylindrical specimens in the present study will thus likely have underestimated the PVF and overestimated the mechanical properties of the composite. 
321 Second, data in the bone group were lost due to slippage of the extensometer upon failure. Application

322 of the extensometer at the center of the specimen is a good practice method, yet for rough surfaces as

323 bone, it might be better to use a larger extensometer attached to the endcaps instead of the bone surface

324 itself [20]. This would overcome the problem of slippage and prevent potential damage caused by the

325 attachment of the extensometer to the surface. Another advantage of this technique is that it measures

326 strain over the entire length of the specimen, rather than sampling at one location on the irregular

327 specimen. In this study, only the central $6 \mathrm{~mm}$ of the specimen was captured for strain measurement. If

328 the deformation took place outside this extensometer gage length, this might have caused less precise

329 measurements of the yield and ultimate strain.

330 Third, the strain rate during the ramp to failure was not perfectly equal to $0.5 \%$ per second. Given the 331 instability of the extensometer measurement towards failure, we chose for displacement control in the 332 ramp to failure. We aimed for a displacement rate that would correspond to a strain rate of approximately $3330.5 \%$ per second, as applied in previous studies $[11,23]$. Yet, the effective strain rate as measured from 334 the extensometer signal was slightly lower ( $0.4 \pm 0.07 \%$ per second $)$ and showed some variation between 335 specimens. However, based on preliminary testing, this variation did not have a large effect on the 336 mechanical properties.

337 Finally, the material properties found in this study depend on several variables of the PMMA bone cement, 338 such as the composition, storage conditions prior to mixing and the method of mixing. The properties 339 might deviate under different conditions.

\section{5. Conclusions}

341 In conclusion, we found that (1) trabecular bone specimens that were highly aligned with the MTD (mean

342 error $<5^{\circ}$ ) could be obtained with a preparation procedure using specimen-specific 3D-printed cutting 343 guides; (2) the compressive modulus, strength and energy to failure of trabecular bone were significantly 
344 increased by cement augmentation; compared to bone cement itself, the proximal femoral bone-cement

345 composites had a higher compressive modulus, but lower strength; (3) the composite modulus was

346 significantly correlated with BV/TV. These results are in contrast with previous findings on human

347 vertebral bone, where the composite was more compliant than bone cement and no correlation was

348 found between BV/TV and the composite modulus. The mechanical properties of bone-cement

349 composites thus cannot simply be applied across different anatomical sites; the site-specific differences

350 in bone density and trabecular alignment should be taken into account. Collectively, the data suggest that

351 at low BV/TV, cement dominates the composite properties, while at high BV/TV, the contribution of bone

352 becomes apparent, revealing a positive relationship between BV/TV and the on-axis compressive 353 modulus.

\section{Acknowledgements}

This work was funded by the Research Foundation Flanders FWO (application number 1S34218N) and the

ESB Mobility Award. We thank Alexander Meynen for his technical support in 3D-printing of the cutting

357 guides and Walter Coudyzer for acquiring the CT scans of the femur heads. We also thank Jo Verbinnen and Kristof Reyniers from the department of Anatomy KU Leuven for collecting and preparing the femoral 359 heads.

\section{References}

[1] J. Charnley, Anchorage of the femoral head prosthesis to the shaft of the femur., J. Bone Joint Surg. Br. 42 B (1960) 28-30. doi:10.1302/0301-620x.42b1.28.

362 [2] R. Vaishya, M. Chauhan, A. Vaish, Bone cement, J. Clin. Orthop. Trauma. 4 (2013) 157-163. doi:10.1016/j.jcot.2013.11.005.

364 [3] A. Sas, E. Tanck, A. Sermon, G.H. van Lenthe, Finite element models for fracture prevention in patients with metastatic bone disease. A literature review, Bone Reports. 12 (2020) 100286. doi:10.1016/j.bonr.2020.100286.

367 [4] M. Taylor, P.J. Prendergast, Four decades of finite element analysis of orthopaedic devices: 
Where are we now and what are the opportunities?, J. Biomech. 48 (2015) 767-778. doi:10.1016/j.jbiomech.2014.12.019.

[5] T.S. Kaneko, H.B. Skinner, J.H. Keyak, Lytic lesions in the femoral neck: Importance of location and evaluation of a novel minimally invasive repair technique, J. Orthop. Res. 26 (2008) 1127-1132. doi:10.1002/jor.20555.

[6] E. Basafa, R.S. Armiger, M.D. Kutzer, S.M. Belkoff, S.C. Mears, M. Armand, Patient-specific finite element modeling for femoral bone augmentation, Med. Eng. Phys. 35 (2013) 860-865. doi:10.1016/j.medengphy.2013.01.003.

[7] P. Varga, J.A. Inzana, J. Schwiedrzik, P.K. Zysset, B. Gueorguiev, M. Blauth, M. Windolf, New approaches for cement-based prophylactic augmentation of the osteoporotic proximal femur provide enhanced reinforcement as predicted by non-linear finite element simulations, Clin. Biomech. 44 (2017) 7-13. doi:10.1016/j.clinbiomech.2017.03.001.

[8] J. Kok, A. Širka, L. Grassi, D.B. Raina, S. Tarasevičius, M. Tägil, L. Lidgren, H. Isaksson, Fracture strength of the proximal femur injected with a Calcium Sulfate/Hydroxyapatite bone substitute, Clin. Biomech. 63 (2019) 172-178. doi:10.1016/j.clinbiomech.2019.03.008.

[9] M. Kinzl, A. Boger, P.K. Zysset, D.H. Pahr, The effects of bone and pore volume fraction on the mechanical properties of PMMA/bone biopsies extracted from augmented vertebrae, J. Biomech. 44 (2011) 2732-2736. doi:10.1016/j.jbiomech.2011.07.028.

[10] A. Boger, M. Bohner, P. Heini, K. Schwieger, E. Schneider, Performance of vertebral cancellous bone augmented with compliant PMMA under dynamic loads, Acta Biomater. 4 (2008) 16881693. doi:10.1016/j.actbio.2008.06.019.

[11] A. Race, K.A. Mann, A.A. Edidin, Mechanics of bone/PMMA composite structures: An in vitro study of human vertebrae, J. Biomech. 40 (2007) 1002-1010. doi:10.1016/j.jbiomech.2006.04.003.

[12] B. Helgason, P. Stirnimann, R. Widmer, A. Boger, S.J. Ferguson, Influence of cement stiffness and bone morphology on the compressive properties of bone-cement composites in simulated vertebroplasty, J. Biomed. Mater. Res. - Part B Appl. Biomater. 101 B (2013) 364-374. doi:10.1002/jbm.b.32847.

[13] E.F. Morgan, T.M. Keaveny, Dependence of yield strain of human trabecular bone on anatomic site, J. Biomech. 34 (2001) 569-577. doi:10.1016/S0021-9290(01)00011-2.

[14] E.F. Morgan, H.H. Bayraktar, T.M. Keaveny, Trabecular bone modulus-density relationships depend on anatomic site, J. Biomech. 36 (2003) 897-904. doi:10.1016/S0021-9290(03)00071-X.

[15] M.I.I. Jofe, T. Takcuchi, W.C. Hayes, Compressive Behavior of human bone-cement composites, (1987).

[16] J.L. Williams, W.J.H. Johnson, Elastic constants of composites formed from PMMA bone cement and anisotropic bovine tibial cancellous bone, J. Biomech. 22 (1989) 673-682. doi:10.1016/00219290(89)90017-1.

[17] C. Öhman, M. Baleani, E. Perilli, E. Dall'Ara, S. Tassani, F. Baruffaldi, M. Viceconti, Mechanical testing of cancellous bone from the femoral head: Experimental errors due to off-axis measurements, J. Biomech. 40 (2007) 2426-2433. doi:10.1016/j.jbiomech.2006.11.020. 
[18] H. Lv, L. Zhang, F. Yang, Z. Zhao, Q. Yao, L. Zhang, P. Tang, Comparison of microstructural and mechanical properties of trabeculae in femoral head from osteoporosis patients with and without cartilage lesions: A case-control study Pathophysiology of musculoskeletal disorders, BMC Musculoskelet. Disord. 16 (2015) 1-10. doi:10.1186/s12891-015-0530-5.

[19] I. Koh, Y. Gombert, C. Persson, H. Engqvist, B. Helgason, S.J. Ferguson, Ceramic cement as a potential stand-alone treatment for bone fractures: An in vitro study of ceramic-bone composites, J. Mech. Behav. Biomed. Mater. 6 (2016) 519-529. doi:10.1016/j.jmbbm.2016.03.027.

[20] B. Helgason, E. Perilli, E. Schileo, F. Taddei, S. Brynjólfsson, M. Viceconti, Mathematical relationships between bone density and mechanical properties: A literature review, Clin. Biomech. 23 (2008) 135-146. doi:10.1016/j.clinbiomech.2007.08.024.

[21] F. Eckstein, M. Matsuura, V. Kuhn, M. Priemel, R. Müller, T.M. Link, E.M. Lochmüller, Sex differences of human trabecular bone microstructure in aging are site-dependent, J. Bone Miner. Res. 22 (2007) 817-824. doi:10.1359/jbmr.070301.

[22] A. Odgaard, Three dimensional methods for quantification of cancellous bone architecture, Bone. 20 (1997) 315-328.

[23] T.M. Keaveny, T.P. Pinilla, P.R. Crawford, D.L. Kopperdahl, A. Lou, Systematic and random errors in compression testing of trabecular bone, J. Orthop. Res. 15 (1997) 101-110.

[24] A. López, G. Mestres, M. Karlsson Ott, H. Engqvist, S.J. Ferguson, C. Persson, B. Helgason, Compressive mechanical properties and cytocompatibility of bone-compliant, linoleic acidmodified bone cement in a bovine model, J. Mech. Behav. Biomed. Mater. 32 (2014) 245-256. doi:10.1016/j.jmbbm.2014.01.002.

[25] N.H. Hart, S. Nimphius, T. Rantalainen, A. Ireland, A. Siafarikas, R.U. Newton, Mechanical basis of bone strength: Influence of bone material, bone structure and muscle action, J. Musculoskelet. Neuronal Interact. 17 (2017) 114-139.

[26] X. Wang, X. Liu, G.L. Niebur, Preparation of On-Axis Cylindrical Trabecular Bone Specimens Using Micro-CT Imaging, in: Trans. ASME, 2004: pp. 122-125.

[27] Z. Wu, A.J. LaNeve, G.L. Niebur, In vivo microdamage is an indicator of susceptibility to initiation and propagation of microdamage in human femoral trabecular bone, Bone. 55 (2013) 208-215. doi:10.1016/j.bone.2013.02.019.

[28] E. Perilli, M. Baleani, C. Öhman, R. Fognani, F. Baruffaldi, M. Viceconti, Dependence of mechanical compressive strength on local variations in microarchitecture in cancellous bone of proximal human femur, J. Biomech. 41 (2008) 438-446. doi:10.1016/j.jbiomech.2007.08.003.

[29] I. Fleps, H. Bahaloo, P. Zysset, S.J. Ferguson, H. Pálsson, B. Helgason, Empirical relationships between bone density and ultimate strength: A literature review, J. Mech. Behav. Biomed. Mater. 110 (2020) 103866. doi:10.1016/j.jmbbm.2020.103866.

[30] E.F. Morgan, G.U. Unnikrisnan, A.I. Hussein, Bone Mechanical Properties in Healthy and Diseased States, Annu Rev Biomed Eng. 20 (2018) 119-143. doi:10.1146/annurev-bioeng-062117-121139.

[31] H.H. Bayraktar, E.F. Morgan, G.L. Niebur, G.E. Morris, E.K. Wong, T.M. Keaveny, Comparison of the elastic and yield properties of human femoral trabecular and cortical bone tissue, J. Biomech. 
449

450

451

452

453

454

455

456

457

458

459

460

[32] M. Kinzl, A. Boger, P.K. Zysset, D.H. Pahr, The mechanical behavior of PMMA / bone specimens extracted from augmented vertebrae : A numerical study of interface properties, PMMA shrinkage and trabecular bone damage, 45 (2012) 1478-1484. doi:10.1016/j.jbiomech.2012.02.012.

[33] A. Bistolfi, R. Ferracini, C. Albanese, E. Vernè, M. Miola, PMMA-based bone cements and the problem of joint arthroplasty infections: Status and new perspectives, Materials (Basel). 12 (2019) 1-16. doi:10.3390/ma12234002.

[34] T. Russo, A. Gloria, R. De Santis, U. D’Amora, G. Balato, A. Vollaro, O. Oliviero, G. Improta, M. Triassi, L. Ambrosio, Preliminary focus on the mechanical and antibacterial activity of a PMMAbased bone cement loaded with gold nanoparticles, Bioact. Mater. 2 (2017) 156-161. doi:10.1016/j.bioactmat.2017.05.002. 